\title{
RESEARCH
}

Open Access

\section{Platelet adhesion on commercially pure titanium plates in vitro III: effects of calcium phosphate-blasting on titanium plate biocompatibility}

Masayuki Nakamura ${ }^{1}$, Hachidai Aizawa', Hideo Kawabata ${ }^{1}$, Atsushi Sato ${ }^{1}$, Taisuke Watanabe ${ }^{1}$, Kazushige Isobe ${ }^{1}$, Yutaka Kitamura', Takaaki Tanaka ${ }^{2}$ and Tomoyuki Kawase ${ }^{3 *}$ (D)

\begin{abstract}
Background: Platelet-rich plasma (PRP) is often used to improve surface biocompatibility. We previously found that platelets rapidly adhere to plain commercially pure titanium (cp-Ti) plates in the absence, but not in the presence, of plasma proteins. To further expand on these findings, in the present study, we switched titanium plates from a plain surface to a rough surface that is blasted with calcium phosphate (CaP) powder and then examined platelet adhesion and activation.

Methods: Elemental distribution in CaP-blasted cp-Ti plates was analyzed using energy-dispersive X-ray spectroscopy. PRP samples prepared from anticoagulated blood samples of six healthy, non-smoking adult male donors were loaded on CaP-blasted cp-Ti plates for $1 \mathrm{~h}$ and fixed for examination of platelet morphology and visualization of PDGF-B and platelet surface markers (CD62P, CD63) using scanning electron microscopy and fluorescence microscopy. Plain SUS316L stainless steel plates used in injection needles were also examined for comparison.

Results: Significant amounts of calcium and phosphate were detected on the CaP-blasted cp-Ti surface. Platelets rapidly adhered to this surface, leading to higher activation. Platelets also adhered to the plain stainless surface; however, the levels of adhesion and activation were much lower than those observed on the CaP-blasted cp-Ti plate.

Conclusions: The CaP-blasted cp-Ti surface efficiently entraps and activates platelets. Biomolecules released from the activated platelets could be retained by the fibrin matrix on the surface to facilitate regeneration of the surrounding tissues. Thus, PRP immersion could not only eliminate surface air bubbles but also improve the biocompatibility of the implant surface.
\end{abstract}

Keywords: Platelet-rich plasma, CD62P, CD63, Titanium, Implant, Calcium phosphates, Blasting

\footnotetext{
*Correspondence: kawase@dent.niigata-u.ac.jp

${ }^{3}$ Division of Oral Bioengineering, Institute of Medicine and Dentistry, Niigata University, Niigata, Japan

Full list of author information is available at the end of the article
}

\section{Springer Open}

(c) The Author(s). 2020 Open Access This article is licensed under a Creative Commons Attribution 4.0 International License, which permits use, sharing, adaptation, distribution and reproduction in any medium or format, as long as you give appropriate credit to the original author(s) and the source, provide a link to the Creative Commons licence, and indicate if changes were made. The images or other third party material in this article are included in the article's Creative Commons licence, unless indicated otherwise in a credit line to the material. If material is not included in the article's Creative Commons licence and your intended use is not permitted by statutory regulation or exceeds the permitted use, you will need to obtain permission directly from the copyright holder. To view a copy of this licence, visit http://creativecommons.org/licenses/by/4.0/. 


\section{Introduction}

Platelet-rich plasma (PRP) is now widely applied in various fields of regenerative medicine. Its clinical application is based on the evidence that platelets concentrated in PRP provide high levels of growth factors along with fibrinogen/fibrin, both of which are involved in the tissue engineering triangle and are expected to function cooperatively in successful tissue regeneration [1]. PRP is often used to treat the implant surface prior to implantation. The primary purpose of this process is to physically remove air bubbles, thus improving biocompatibility [2]. Other purposes include functionalization of the implant surface with the biomolecules included in PRP preparations. However, no rigorous evidence has emerged to endorse the latter purpose yet.

In the past two decades, surface modification has been the main target of research and development on implant fabrication. To enlarge the surface free area for bone and osteoblast adhesion and thereby reinforce the initial stability of the implants, the implant surface is usually roughened using chemical, electrochemical, and/or mechanical techniques [3]. Among these techniques, blasting is a relatively simpler and more convenient technique, and thus has been applied widely in the implant manufacturing industry. Ishikawa et al. first reported that blasting with hydroxyapatite (HA) powders for the surface modification of implants efficiently coats HA on the titanium (Ti) implant surface [4]. The blasting technique used in this study adopts calcium phosphate $(\mathrm{CaP})$ powder $(\mathrm{HA}: \beta$-tricalcium phosphate $=1: 4$ ) as an abrasive $[5,6]$. The attachment and retention of $\mathrm{CaP}$ on the Ti surface improves its biocompatibility and induces osseointegration by virtue of its high osteoconductivity [7-10].

Till date, many studies have investigated osteoblast behavior on $\mathrm{Ti}$ surfaces [11-14]. Judging from the low blood compatibility, it is predictable that platelets are activated to attach and aggregate on the Ti surface. In case of non-anticoagulated blood, it is believed that fibrin networks are formed on the Ti surface to induce the adhesion of platelets initially, followed by osteoblasts or mesenchymal stem cells next $[3,15]$. This process is thought to significantly influence osseointegration and the subsequent mineralization process. In previous studies $[2,16]$, we examined platelet behavior on the plain cp-Ti surface in case of anticoagulated blood and found that the adhesion of platelets served in the form of PRP onto the cp-Ti surface is significantly suppressed by plasma proteins, such as albumin. Thus, we concluded that PRP immersion may not be as helpful as expected in the functionalization of the implant surface. However, this conclusion is based on the data obtained from plain cp-Ti surfaces, and it is possible that platelets may act differentially on a rough surface. The rough surface model used in the present study mimics a certain type of implant commercially available in the clinical setting.

To expand our previous findings, in the present study, we examined and compared platelet adhesion and activation on CaP-blasted cp-Ti and plain SUS316L stainless steel plates. The plain SUS316L stainless steel plates were used as a model of injection needles used for blood collection and subsequent PRP preparation. Since both $\mathrm{CaP}$-coating and roughness increase protein adsorption and cell adhesion $[17,18]$, we initially raised a working hypothesis that the $\mathrm{CaP}$-blasted $\mathrm{cp}$-Ti plate may capture and retain a greater number of platelets, even in the form of liquid PRP, than the plain $\mathrm{cP}$-Ti plate. However, although albumin and other plasma components are present in the form of PRP, the ability of CaP-blasted $\mathrm{cp}-\mathrm{Ti}$ plates to trap and retain platelets was much higher than expected.

\section{Materials and methods Preparation of pure PRP}

Blood samples were collected from six non-smoking, healthy, male volunteers aged 46-62 years. Despite having lifestyle-related diseases and taking medication, these donors, that is, our team members and our relatives, had no limitations on the activities of daily living. These donors were also declared to be free of HIV, HBV, HCV, or syphilis infections. In addition, a prothrombin test was performed on all the blood samples by means of CoaguChek $^{\oplus}$ XS (Roche, Basel, Switzerland), and all the samples were found to be normal. The study design and consent forms for all the procedures (project identification code: 2297) were approved by the Ethics Committee for Human Participants at the Niigata University School of Medicine (Niigata, Japan) and complied with the Helsinki Declaration of 1964, as revised in 2013.

First, $\sim 9 \mathrm{~mL}$ of peripheral blood was collected in plain glass vacuum blood collection tubes (Vacutainer; ${ }^{\oplus}$ Beckton \& Dickinson, Franklin Lakes, NJ, USA) containing $1.5 \mathrm{~mL}$ of a formulation of acid-citrate-dextrose (ACDA) $[2,16]$. Whole-blood samples were stored in a rotating agitator at ambient temperature and were used within $24 \mathrm{~h}$. Thereafter, the samples were centrifuged at $402 \times g$ for $8 \mathrm{~min}$ (soft spin). The upper plasma fraction, $\sim 2 \mathrm{~mm}$ beyond the interface between the plasma and red blood cell fractions, was then transferred into 2$\mathrm{mL}$ sample tubes and centrifuged once again at $1065 \times g$ for $3 \mathrm{~min}$ (hard spin) to collect the resting platelet pellets. Afterwards, the platelets were resuspended in acellular plasma to adjust the platelet concentration to $3.0 \times 10^{5} / \mu \mathrm{L}$.

Platelets and other blood cell counts were measured using a pocH 100iV automated hematology analyzer (Sysmex, Kobe, Japan). The exclusion of white and red 
blood cells was confirmed in pure PRP preparations prior to the following experiments.

\section{CaP-blasted cp-Ti plates and platelet inoculation}

cp-Ti plates (Nilaco, Tokyo, Japan) were blasted with a mixture of HA and $\beta$-TCP (in a ratio of 1:4) at $0.3 \mathrm{MPa}$. The resulting cp-Ti plates were cut into small, square 10 $\times 10 \mathrm{~mm}^{2}$ pieces; washed serially with acetone $(60 \mathrm{~s})$, ethanol $(2 \times 60 \mathrm{~s})$, and distilled water $(2 \times 60 \mathrm{~s})$ in an ultrasonic cleaner (Citizen, Tokyo, Japan); and air-dried.

Platelet suspensions $\left(3 \times 10^{7}\right.$ per plate $)$ were inoculated onto CaP-blasted plates and the plates were incubated at ambient temperature for $60 \mathrm{~min}$. The CaPblasted plates were then vigorously washed two times with phosphate-buffered saline (PBS) on a shaker ( $10 \mathrm{~s})$ and subjected to spectrophotometric assay without fixation or with $10 \%$ neutralized formalin fixation for staining. For energy dispersive X-ray spectroscopy (EDS) (JCM-6000 with JED-2300; JEOL, Akishima, Japan) and scanning electron microscopy (SEM) (TM1000; Hitachi, Tokyo, Japan) examination, the samples were fixed with $2.5 \%$ glutaraldehyde and dehydrated serially, as described below.

Since we vigorously examined plain cp-Ti plates in the previous studies [2, 16], we adopted plain SUS-316 stainless steel plates (Nilaco) for comparison with the CaP-blasted cp-Ti plates. This choice was based on the routine preparation of PRP by many clinicians and operators in private practice using syringes equipped with needles usually composed of SUS316L. To evaluate platelet loss and activation in the preparation process, immersion in PRP and examination of adherent platelets were performed, as detailed below.

\section{Plate examination using SEM}

To evaluate their surface roughness, both plate types were examined via SEM and atomic force microscopy (AFM). The surfaces of the plates were coated with gold-palladium using a sputter coater (MSP-1S; Vacuum Device, Mito, Japan) and examined using SEM (Hitachi) operated at an accelerating voltage of $15 \mathrm{kV}[11,19]$. To examine platelet adhesion and morphology, the platelets adhering to the plates were fixed with $2.5 \%$ neutralized glutaraldehyde, dehydrated, and freeze-dried as described previously [20,21].

\section{Plate examination using AFM}

To evaluate the surface roughness, both plate types were directly subjected to AFM examination. The AFM height images of the samples were recorded in alternating current (AC) mode at room temperature in PBS using the NanoWizard 3 (JPK Instruments AG, Berlin, Germany) AFM system. Soft cantilevers (contact-G;
BudgetSensors, Sofia, Bulgaria) were used for scanning through the quantitative imaging mode $[2,11]$.

\section{Elemental examination and mapping using EDS}

For elemental analysis and mapping of CaP-blasted $\mathrm{Ti}$ plates, platelet samples were prepared, fixed, dehydrated, and sputter-coated with Au-Pd (vacuum device) using SEM with an EDS detector (JEOL) at an acceleration voltage of $10 \mathrm{kV}$. Cell-free samples were directly sputter-coated with gold-palladium and used for EDS examination. The data provided the elemental composition from the surface to a depth of $1 \mu \mathrm{m}$ under the conditions recommended by the manufacturer [11].

\section{Quantitative and qualitative determination of adherent platelet counts}

Platelets were inoculated onto the surface of CaP-blasted $\mathrm{Ti}$ and plain SUS316 plates and incubated for $60 \mathrm{~min}$. The plates were then washed with PBS to remove nonadherent platelets and were further incubated for $2 \mathrm{~h}$ with a highly water-soluble tetrazolium dye (Cell Counting Kit-8; Dojindo, Kumamoto, Japan). After incubation, $100 \mu \mathrm{L}$ of the supernatant was collected and its absorbance was measured using a microplate reader (Model 680; Bio-Rad, Hercules, CA, USA) at a wavelength of $450 \mathrm{~nm}$ (reference: $570 \mathrm{~nm}$ ) [2].

Alternatively, platelets were fixed with $10 \%$ neutralized formalin, microperforated with $0.1 \%$ Tween-20containing PBS (T-PBS) for $1 \mathrm{~min}$, and stained with phalloidin (Cytopainter Phalloidin-iFlour 555 Reagent; Abcam, Cambridge, MA, USA) at ambient temperature in the dark and observed under a fluorescence microscope (ECLIPSE 80i; Nikon, Tokyo, Japan) connected to a cooled CCD camera (VB-7000; Keyence, Osaka, Japan). The occupancy of adherent platelets was determined using image analysis software (WinROOF version 6.0, Mitani Corp., Fukui, Japan). In brief, three images were randomly selected from each sample. After RGB separation and manually optimizing the threshold, the images were binarized [19], and the apparent areas covered by platelets per region of interest were calculated. The data were obtained from a single representative experiment.

\section{Immunocytochemical fluorescence staining}

The CaP-blasted $\mathrm{Ti}$ and plain SUS316L plates were washed with $\mathrm{PBS}$ and the fixed platelets were microperforated with T-PBS (0.1\% Tween-20-containing PBS) for 1 min [16]. The samples were washed twice with PBS and blocked with $0.1 \%$ Block Ace (Sumitomo Dainippon Pharma Co. Ltd., Osaka, Japan) in T-PBS for $1 \mathrm{~h}$. The samples were then treated with mouse monoclonal antiCD62P, anti-CD63 (1:100 dilution; BioLegend, San Diego, CA, USA), or anti-PDGF-B (1:200 dilution; Santa Cruz Biotechnology, Dallas, TX, USA) antibodies 
overnight at $4{ }^{\circ} \mathrm{C}$. Post-treatment, the samples were again washed twice with T-PBS and subsequently probed with a secondary antibody (goat anti-mouse IgG H\&L conjugated with Alexa Fluor ${ }^{\circ}$ 488; Abcam, Cambridge, MA, USA) for $60 \mathrm{~min}$ along with phalloidin (Cytopainter Phalloidin-iFlour $^{\text {tw }} 555$ Reagent; Abcam) at ambient temperature in the dark. Isotype controls for mouse primary antibodies (Abcam) were used as negative controls.

Finally, after washing with PBS, the samples were mounted using an antifade mounting medium (Vectashield $^{\oplus}$; Vector Laboratories, Burlingame, CA, USA), and target proteins were examined under a fluorescence microscope (Eclipse 80i; Nikon, Tokyo, Japan) connected to a cooled CCD camera (VB-7000; Keyence, Osaka, Japan) [22].

\section{Visualization of polyphosphates}

Polyphosphates stored in platelets and released from platelets were visualized using 4',6-diamidino-2-phenylindole (DAPI; Dojindo, Kumamoto, Japan). PRP was loaded on the plates, as described above, washed, and fixed with $10 \%$ neutralized formalin for $30 \mathrm{~min}$. The platelets were then treated with $0.01 \mathrm{~g} / \mathrm{mL}$ DAPI- and phalloidin-containing PBS for $15 \mathrm{~min}$ and subjected to microscopic examination using a fluorescence microscope (Eclipse 80i; Nikon) equipped with a BV-2A filter cube (excitation filter: $400-440 \mathrm{~nm}$; dichroic mirror: 455 $\mathrm{nm}$; barrier filter: $470 \mathrm{~nm}$ ) and a G-2A filter cube (excitation filter: $510-560 \mathrm{~nm}$; dichroic mirror: $575 \mathrm{~nm}$; barrier filter: $590 \mathrm{~nm}$ ) to detect DAPI and phalloidin, respectively.

\section{Contact angle analysis}

The contact angles of the CaP-blasted $\mathrm{cp}$ - $\mathrm{Ti}$ and SUS316L platelets were measured using a contact angle meter (LSE-ME1; Nick Corp., Kawaguchi, Japan) to determine the time-course changes in the surface wettability [11]. Each plate was cleaned with acetone, rinsed with ethanol, dried as described above, and subjected to contact angle analysis using distilled water for up to $15 \mathrm{~min}$.

\section{Statistical analysis}

Data are expressed as the mean \pm standard deviation. For two-group comparisons, a Mann-Whitney rank sum test was performed to compare mean values (SigmaPlot 13.0; Systat Software, Inc., San Jose, CA, USA). For multigroup comparisons, according to the suggestion based on the results of both normality and equal variance testing, a one-way ANOVA followed by Bonferroni's multiple-comparisons test was performed to compare the mean values (SigmaPlot 13.0). $P<0.05$ was considered statistically significant.
G*Power software (version 3.1.9.7. Heinrich-HeineUniversität Düsseldorf, Düsseldorf, Germany) was used to calculate the power of the statistical analyses and confirm the validity of the statistical analyses. For the data shown in Figs. 6 and 8, the power values were 1.00.

\section{Results}

Figure 1 shows the surface roughness of CaP-blasted cp$\mathrm{Ti}$ and plain SUS316L plates. The surface of the CaPblasted $\mathrm{cp}$-Ti plate was substantially rough, whereas that of the SUS316L plate was only roughened slightly. The maximum heights (from the bottom of concave to the top of convex) in these images were approximately 2.3 $\mu \mathrm{m}$ (CaP-blasted cp-Ti) and $350 \mathrm{~nm}$ (SUS316L).

Figure 2 shows time-course changes in the contact angles of CaP-blasted cp-Ti and SUS316L surfaces. For up to $15 \mathrm{~min}$, the contact angles of both the plates declined from approximately 82 to 63 [CaP-blasted cp-Ti: $82.5 \pm$ $3.2^{\circ}(10 \mathrm{~s}), 79.7 \pm 4.1^{\circ}(2 \mathrm{~min}), 77.1 \pm 4.9^{\circ}(5 \mathrm{~min}), 71.8$ $\pm 5.2^{\circ}(10 \mathrm{~min}), 65.6 \pm 5.8^{\circ}(15 \mathrm{~min})$ ] [SUS316L: $82.6 \pm$ $5.6^{\circ}(10 \mathrm{~s}), 80.4 \pm 5.8^{\circ}(2 \mathrm{~min}), 76.9 \pm 6.3^{\circ}(5 \mathrm{~min}), 70.2$ $\left.\pm 7.1^{\circ}(10 \mathrm{~min}), 61.5 \pm 8.5^{\circ}(15 \mathrm{~min})\right]$ : similarly in a time-dependent manner, and significant differences between these plates were not observed at any of the time points.

Figure 3 shows the elemental mapping of the surfaces of plain and CaP-blasted cp-Ti plates. Because the plain cp-Ti plate was not chemically or mechanically mirrorfinished, relatively large (micron order) yet gentle roughness was observed on the surface. In addition, Ti (indicated by blue dots) was detected as a major element on the surface. After CaP-blasting, sub-micron levels of roughness were formed on the surface, and some areas were coated with $\mathrm{Ca}$ (red) and P (green). Under these blasting conditions, the area ratio of $\mathrm{Ti}$ to $\mathrm{CaP}$ was approximately 50:50. In addition, after incubation with PRP for $60 \mathrm{~min}$, the platelets adhered to the surface mainly by means of their pseudopodia, regardless of the distribution of surface elements or topography (convex or concave).

Figure 4 shows the elemental analysis of the plain and $\mathrm{CaP}$-blasted cp-Ti plate surfaces. In the plain $\mathrm{cp}$-Ti surface used for blasting, $\mathrm{Ti}$ was the only major element among the elements tested (Ti: approximately 8000, Ca: $<100$, P: $<100$ counts) (Fig. 2a). CaP-blasting increased $\mathrm{Ca}$ and P contents (Ca: approx. 2700, P: 2800 counts), but reduced apparent Ti contents (approx. 5000 counts), and the ratios of $\mathrm{Ca}$ and $\mathrm{P}$ to $\mathrm{Ti}$ were roughly 0.54 and 0.56 , respectively (Fig. 2b). Due to the adhered platelets, adsorbed plasma proteins, and other components on the CaP-blasted cp-Ti surface, incubation with PRP substantially reduced the apparent counts of these elements ( $\mathrm{Ti}$ : approx. 230, Ca: 80, P: 70 counts) (Fig. 2c). Nevertheless, compared with the non-blasted cp-Ti surface (Fig. 2a), 

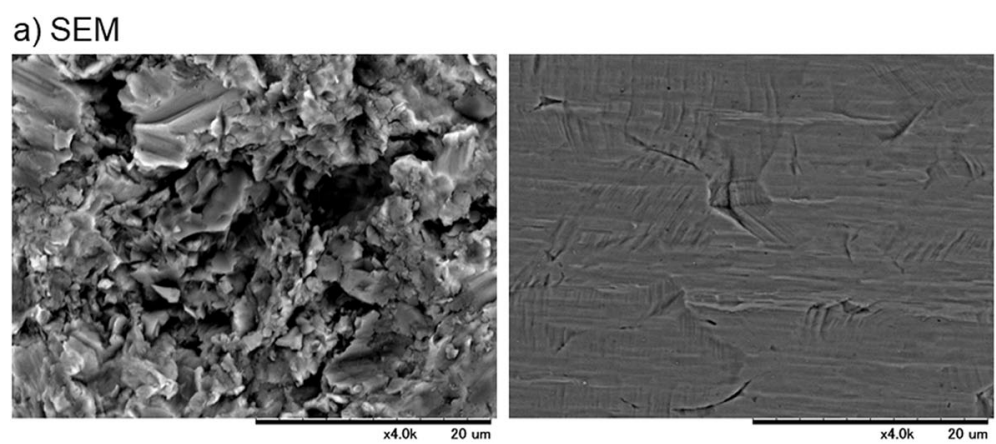

b) AFM (height image)
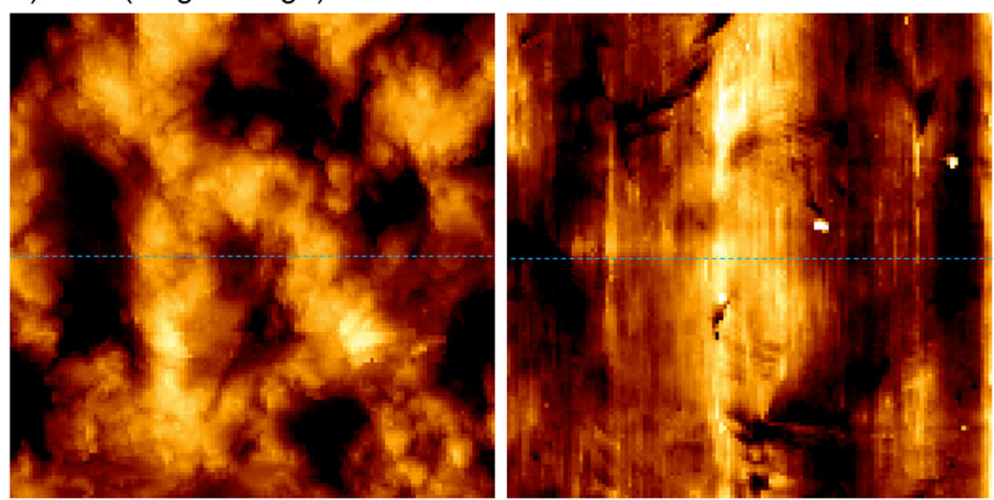

c) AFM (roughness curve)

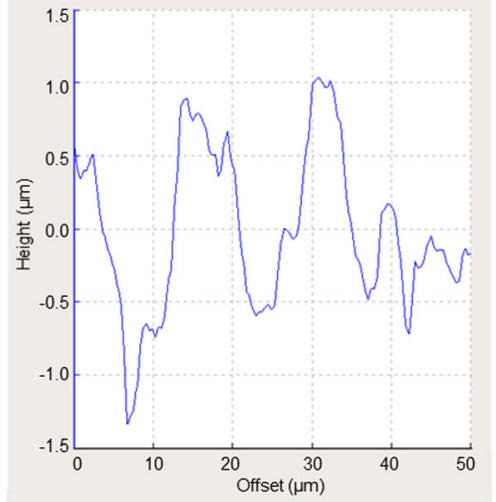

CaP-blasted cp-Ti

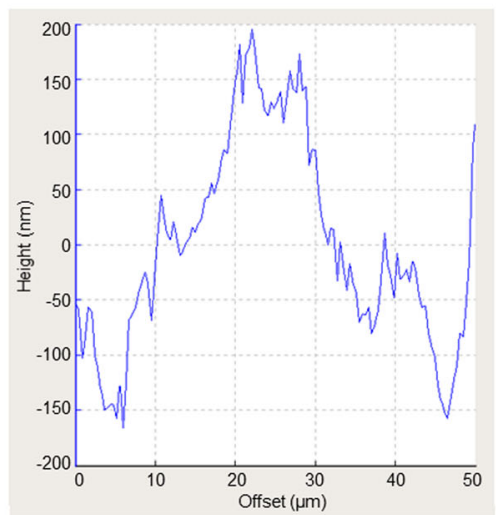

Plain SUS316L

Fig. 1 Surface microstructure of CaP-blasted cp-Ti and plain SUS316L plates. a The plates were directly sputtered without fixation or dehydration process and subjected to SEM examination. b The plates were directly subjected to AFM examination. Size of the height images is $50 \times 50 \mu \mathrm{m}$. $\mathbf{c}$ Roughness profiles were drawn from the height images (blue dashed lines)

the ratios of the elements $(\mathrm{Ca} / \mathrm{P}: 0.34, \mathrm{P} / \mathrm{Ti}: 0.30)$ seemed similar to those on the same plate without incubation with PRP. It is noted that PRP with relatively low platelet counts were used to avoid full coverage of the surface by platelets.

Figure 5 shows the time- and count-dependent platelet adhesion on CaP-blasted cp-Ti plates. When platelets were inoculated on the plates at a count of $3 \times 10^{7} /$ plate, platelet adhesion increased in a time-dependent manner. After $60 \mathrm{~min}$ of incubation, the plate was almost fully covered by platelets. When compared at $20 \mathrm{~min}$ of incubation, platelet adhesion increased in a platelet-countdependent manner. At a count of $9 \times 10^{7} /$ plate, the plate was almost fully covered by platelets.

Figure 6 shows the time- and count-dependent platelet occupancy on CaP-blasted cp-Ti plates. The number of platelets adhered on CaP-blasted cp-Ti plates increased with time (20 min: $36.8 \pm 2.1 \%, 40 \mathrm{~min}: 70.0 \pm 6.0 \%, 60$ min: $83.4 \pm 2.2 \%)$ and inoculum size $\left(3 \times 10^{7} /\right.$ plate: 37.5 $\pm 5.1 \%, 6 \times 10^{7} /$ plate: $49.7 \pm 3.6 \%, 9 \times 10^{7} /$ plate: $78.1 \pm$ 


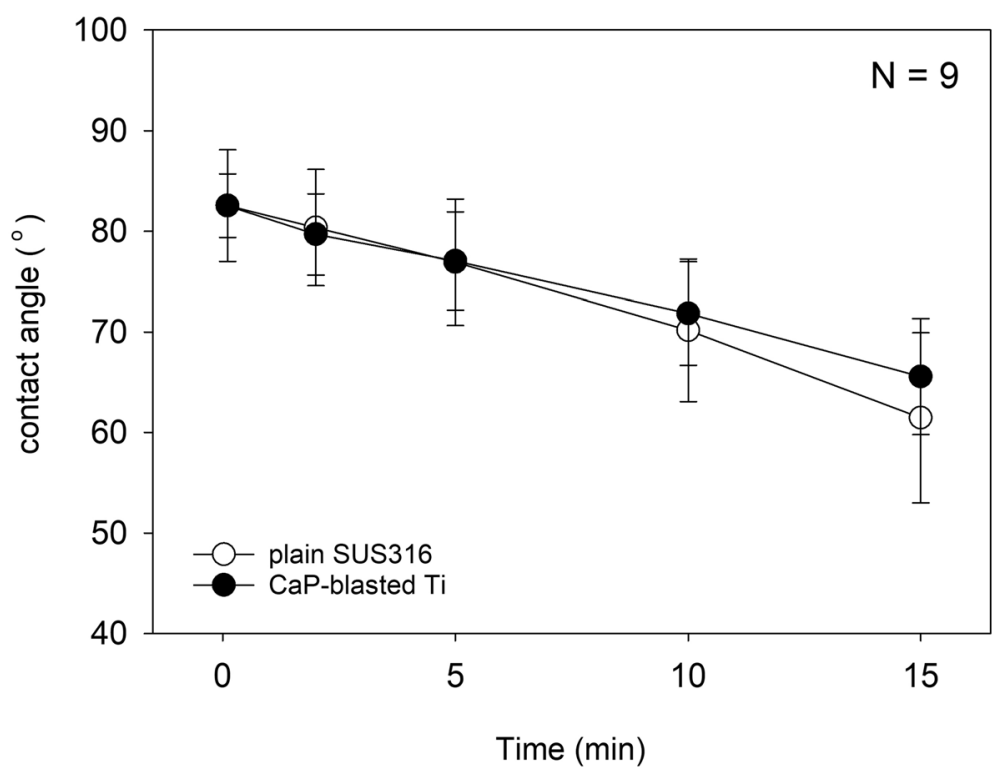

Fig. 2 Time-course changes in contact angles of CaP-blasted cp-Ti and SUS316L surfaces. There was no statistical difference between these surfaces at any of the time points. $N=9$

$2.1 \%)$. The images that the plates were almost fully covered by platelets were evaluated by the image analysis to be approximately $80 \%$ occupancy.

Hereafter, the data are expressed by comparison with plain SUS316L plates, which are widely used in the production of medical devices, including injection needles. For reference, platelet adhesion and reaction on plain cp-Ti plates have been vigorously reported in previous studies [2, 16]. Platelet density and morphology were examined using SEM at low and high magnification. Figure 7 shows SEM observations of platelets adhered on CaP-blasted cp-Ti and plain SUS316L plates after 60min incubation. In CaP-blasted cp-Ti plates, when PRP with relatively high platelet counts was used (vs. Fig. 3), platelets adhered to both the convex and concave surfaces of the micro-topography at higher densities. Some platelets formed pseudopodia; however, the majority of adherent platelets were spherical. In contrast, on plain SUS316L plates, platelet density was seemingly much lower than that on CaP-blasted cp-Ti plates, and many platelets adhered onto the surface by spreading their pseudopodia.

To confirm the difference in platelet density, platelet counts were quantified using a tetrazolium salt. Figure 8 shows the number of platelets adherent on CaP-blasted cp-Ti and plain SUS316L plates. Because we prepared pure PRP, significant numbers of white and red blood cells were excluded from the resulting pure PRP preparations. Even though some white and/or red blood cells were included, such contamination is not believed to significantly disturb platelet counts. The spectrophotometric assay demonstrated that considerably greater levels of platelets were entrapped and adherent on the CaP-blasted cp-Ti plates $\left(A_{450 / 570}: 2.373 \pm 0.187\right)$, as compared to plain SUS316L plates $\left(A_{450 / 570}: 0.037 \pm 0.019\right)(P<0.05)$.

Figure 9 shows immunocytochemical detection of activated platelets that express $\mathrm{CD} 62 \mathrm{P}$ and $\mathrm{CD} 63$. In the negative control (using non-immunized mouse IgG), faint non-specific detection was observed: however, this level was substantially lower than that required for specific detection. The expression levels of both CD62P and CD63 (green) were substantially upregulated in individual platelets (which were marked by cytoskeletal actin fibers shown by red) on CaP-blasted cp-Ti plates, as compared to plain SUS316L plates. This implies that platelets adherent on CaP-blast cp-Ti plates are activated at higher levels.

Since activated platelets release biomolecules, such as growth factors, the representative growth factors PDGF-B and polyphosphates were subsequently visualized. Figure 10 shows the immunocytochemical visualization of PDGF-B and the chemical detection of polyphosphates. On CaP-blasted cp-Ti plates, PDGF-B was detected mainly in extra-platelet spaces, whereas this growth factor was colocalized with cytoplasmic actin fibers or found in extra-platelet spaces on plain SUS316L plates. In contrast, DAPI-reactive particles (green), that is, polyphosphates, were found in platelets on plain SUS316L plates, while DAPIreactive polyphosphates were widely diffused in the form of smears within extra-platelet spaces (faintly stained by green). 

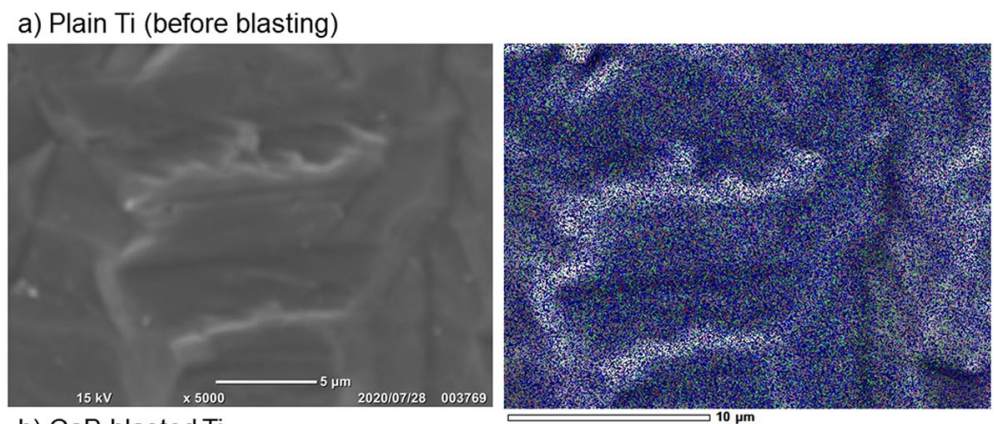

b) CaP-blasted Ti
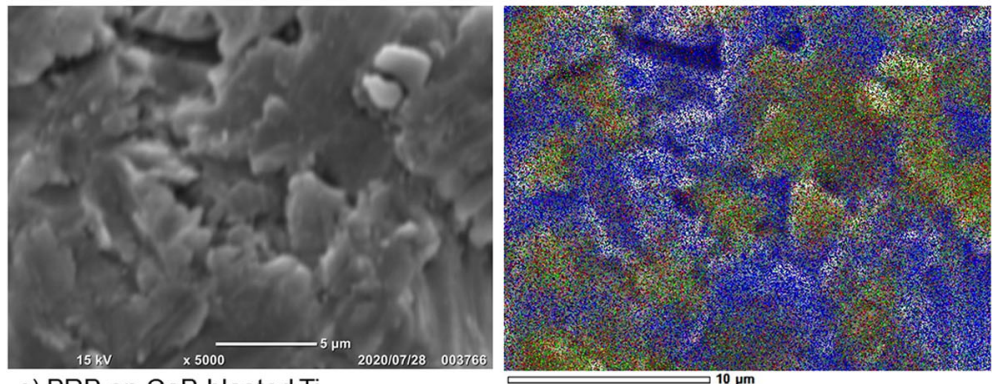

c) PRP on CaP-blasted Ti

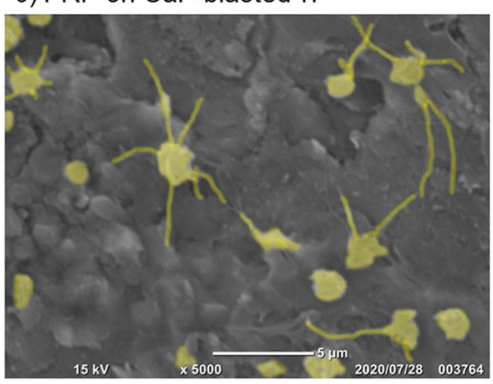

SEM

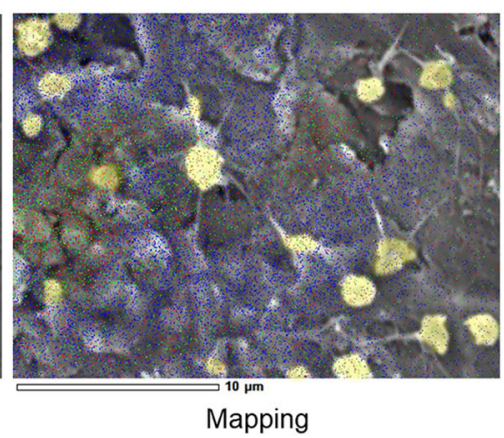

Fig. 3 Elemental mapping of plain and CaP-blasted cp-Ti plate surfaces. The a plain and $\mathbf{b}$ CaP-blasted cp-Ti plates were directly subjected to mapping. c Alternatively, after treatment with PRP for $60 \mathrm{~min}$, the plates with adherent platelets were fixed, dehydrated, and examined using EDS. Blue, red, and green dots represent $\mathrm{Ti}, \mathrm{Ca}$, and $\mathrm{P}$, respectively. Adherent platelets are faint yellow

\section{Discussion}

This study demonstrated that CaP-blasted cp-Ti plates are more efficient in the entrapment and activation of platelets compared to plain $\mathrm{cp}-\mathrm{Ti}$ plates examined in previous studies $[2,16]$. Thus, CaP-blasted Ti implants could be functionalized with platelets and biomolecules provided by PRP to contribute to more significant regeneration of surrounding tissues and consequent osseointegration. Although further studies are required, such as to validate these findings in animal models, the present study provides evidence that supports the PRP immersion of implants prior to application in clinical settings and suggests that as necessity, the period of immersion time can be modulated by platelet counts in individual PRP preparations (for a more detailed discussion, see the subsection "Clinical Relevance").

\section{Platelet adhesion and activation}

The differences in platelet adhesion between CaP-blasted cp-Ti and plain SUS316L plates were speculated to be due to variations in surface wettability, energy, roughness, and/or chemical composition [15, 23]. As reported previously [5], CaP-blasting enlarges the free surface area, increases the surface roughness, and enables the attachment of $\mathrm{CaP}$ (which serves as an abrasive). Among these factors, chemical composition is the well-known to influence protein adsorption. In general, $\mathrm{CaP}$ surfaces adsorb a greater amount of protein than $\mathrm{Ti}$ surfaces [24]. Therefore, it could be hypothesized that platelets adhere more efficiently to the surface of CaP-blasted cp-Ti plates. As expected, we found that a greater number of platelets were entrapped on the surface of CaP-blasted cp-Ti plates than on the surface of plain cp-Ti plates (as well as plain SUS316L plates), even when treated in the form of PRP. 


\section{a) Plain Ti (before blasting)}

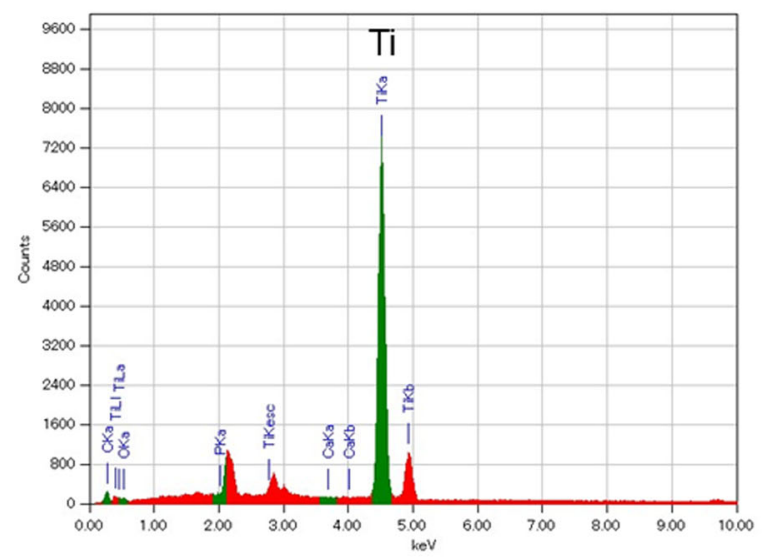

b) CaP-blasted Ti

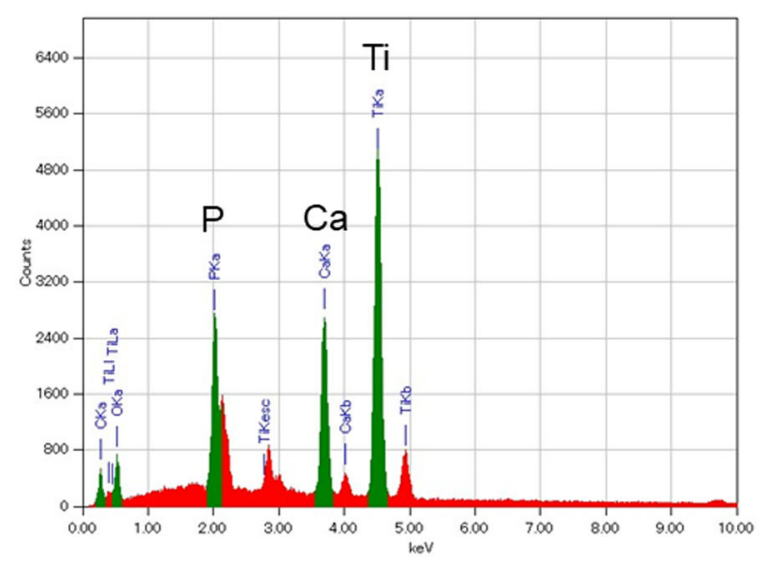

c) PRP on CaP-blasted Ti

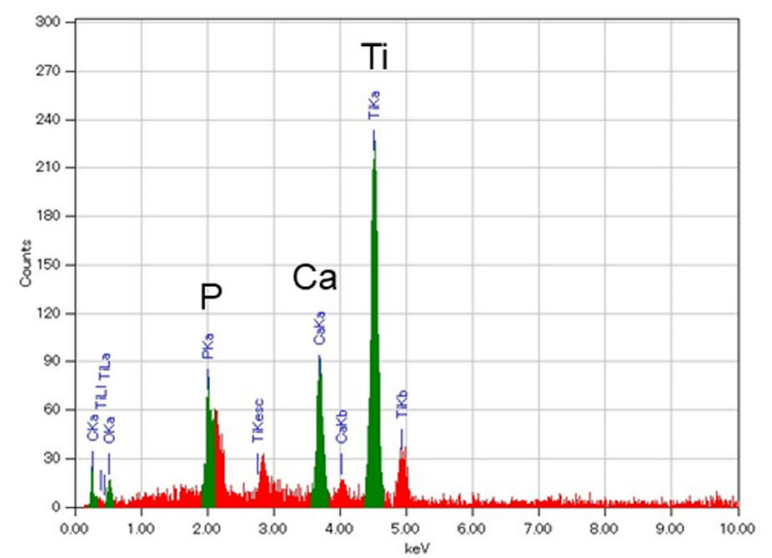

Fig. 4 Elemental analysis of plain and CaP-blasted cp-Ti plate surfaces. a Plain $\mathrm{cp}$-Ti plate before blasting, $\mathbf{b}$ CaP-blasted $\mathrm{cp}$-Ti plate, and c PRP-treated, CaP-blasted cp-Ti plate

However, since we preliminarily observed that platelets were entrapped at much lower levels on decalcified blasted cp-Ti plates, i.e., CaP-free, blasted cp-Ti plates, the efficient entrapment may not be solely due to the specific surface micro-topography or enlarged surface area. This finding is consistent with that of a previous study [25]. However, it cannot be ruled out that decalcification with EDTA may also influence surface chemistry and/or wettability and that micro-topography may function synergistically with $\mathrm{CaP}$-coating, as described below.

We also examined other factors in this study, such as wettability, and found no significant differences between the CaP-blasted cp-Ti and plain SUS316L plates. This observation could be explained by the data described below. $\mathrm{CaP}$ and $\mathrm{HA}$, alone or in combination, display high wettability (contact angle: 40-60 ) [26], and the contact angle of $\mathrm{cp}-\mathrm{Ti}$ is $55-60^{\circ}$ [27]. In addition, despite the use of a $\mathrm{Ti}$ alloy $(\mathrm{Ti}-6 \mathrm{Al}-4 \mathrm{~V})$, surface roughness has a weak impact on wettability [28].

Recently, numerous studies have vigorously investigated the adsorption of proteins on $\mathrm{CaP}$ surfaces [29-32]. In a previous study [2], we demonstrated the involvement of adhesion molecules adsorbed on plain $\mathrm{cp}$ - Ti plates in platelet adhesion. Thus, adhesion molecules, such as fibronectin and von Willebrand factor, may potentially get adsorbed at higher levels on $\mathrm{CaP}$-blasted $\mathrm{cp}$ - $\mathrm{Ti}$ surfaces than on plain surfaces, which allows CaP-blasted cp-Ti surfaces to gather platelets more efficiently. However, it still remains to be clarified the reason why other plasma proteins, such as albumin, do not disturb the platelet adhesion, as observed on the plain surface [2]. Thus, further investigation is needed to clarify the mechanisms responsible for enhanced cell adhesion and protein adsorption on the CaP-blasted cp-Ti surfaces, as compared to plain surfaces.

Platelets are naïve cells that can be easily activated by various mechanical and chemical stimuli, such as centrifugation, and contact with low-biocompatible materials, fibrin, and adenosine diphosphate. In previous studies $[2,16]$, we demonstrated that platelets served in the form of PBS suspension are significantly activated to upregulate $\mathrm{CD} 62 \mathrm{P}$ and $\mathrm{CD} 63$ expression and growth factor release on the $\mathrm{CaP}$-blasted $\mathrm{cp}$ - $\mathrm{Ti}$ surface, compared to on the plain cp-Ti surface. Thus, although the mechanisms for this effect are not fully understood, it is evident that adhesion by itself activates platelets and potentially vice versa.

Differences in surface topography render it difficult to compare the levels of platelet activation between plain and $\mathrm{CaP}$-blasted $\mathrm{cp}$-Ti plates simply by means of image analysis. However, platelets seemed to be more activated on the CaP-blasted cp-Ti surface, rather than on the plain surface. This may be due to enhanced platelet adhesion on the CaP-blasted cp-Ti surface.

\section{Biomolecule release and entrapment in extra-platelet spaces}

It is well known that activated platelets degranulate $\alpha$ and dense granules [33]. In this study, PDGF-B (and 

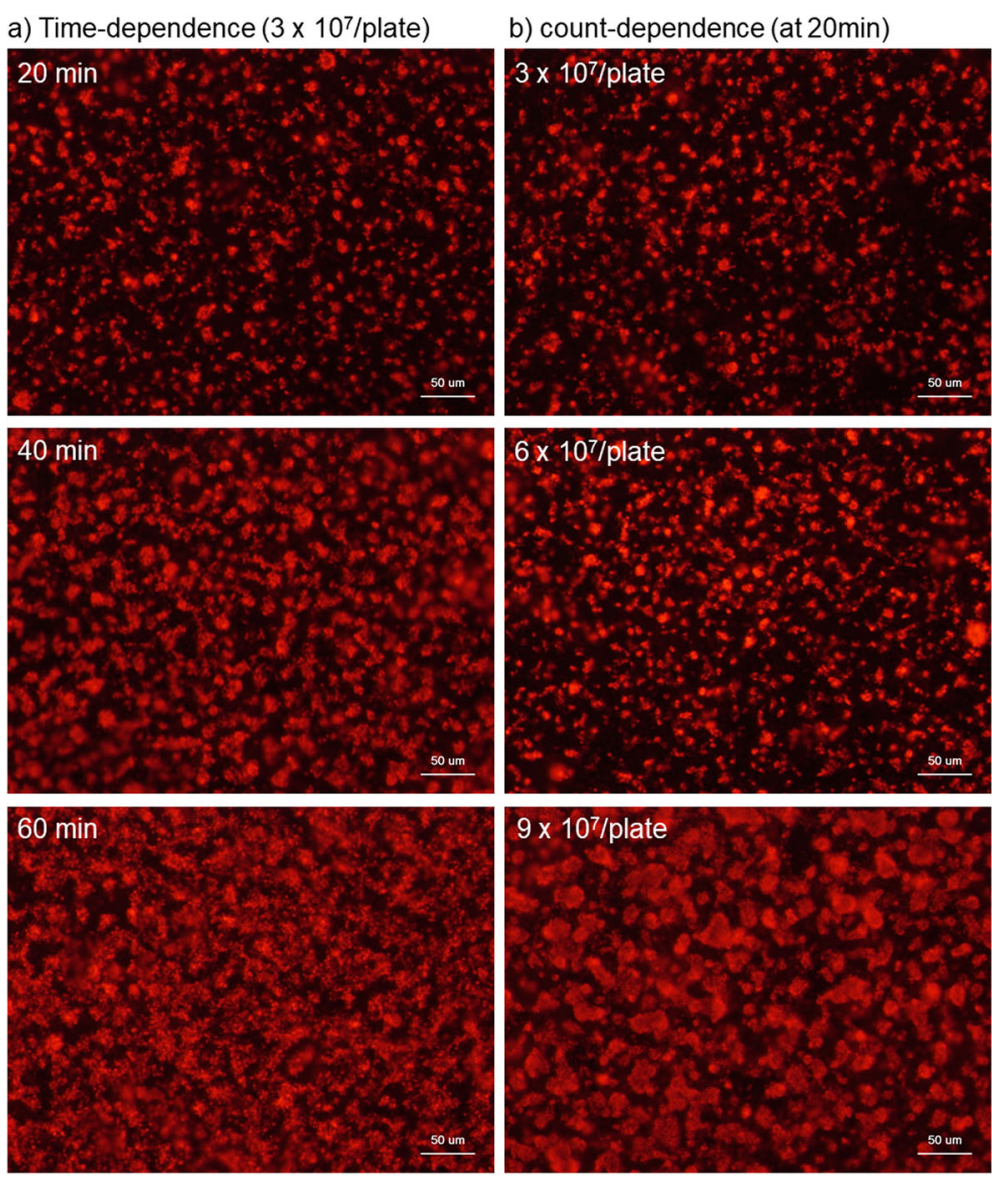

Fig. 5 Time- and count-dependent platelet adhesion on CaP-blasted cp-Ti plates. a Platelets in the form of PRP was inoculated at the fixed count of $3 \times 10^{7}$ /plate and incubated at ambient temperature for 20,40, and $60 \mathrm{~min}$. b Platelets in the form of PRP was inoculated at counts of $3 \times, 6$ $\times$, and $9 \times 10^{7} /$ plate and incubated for the fixed period of time, $20 \mathrm{~min}$. Cytoskeletal actin filaments were stained with phalloidin
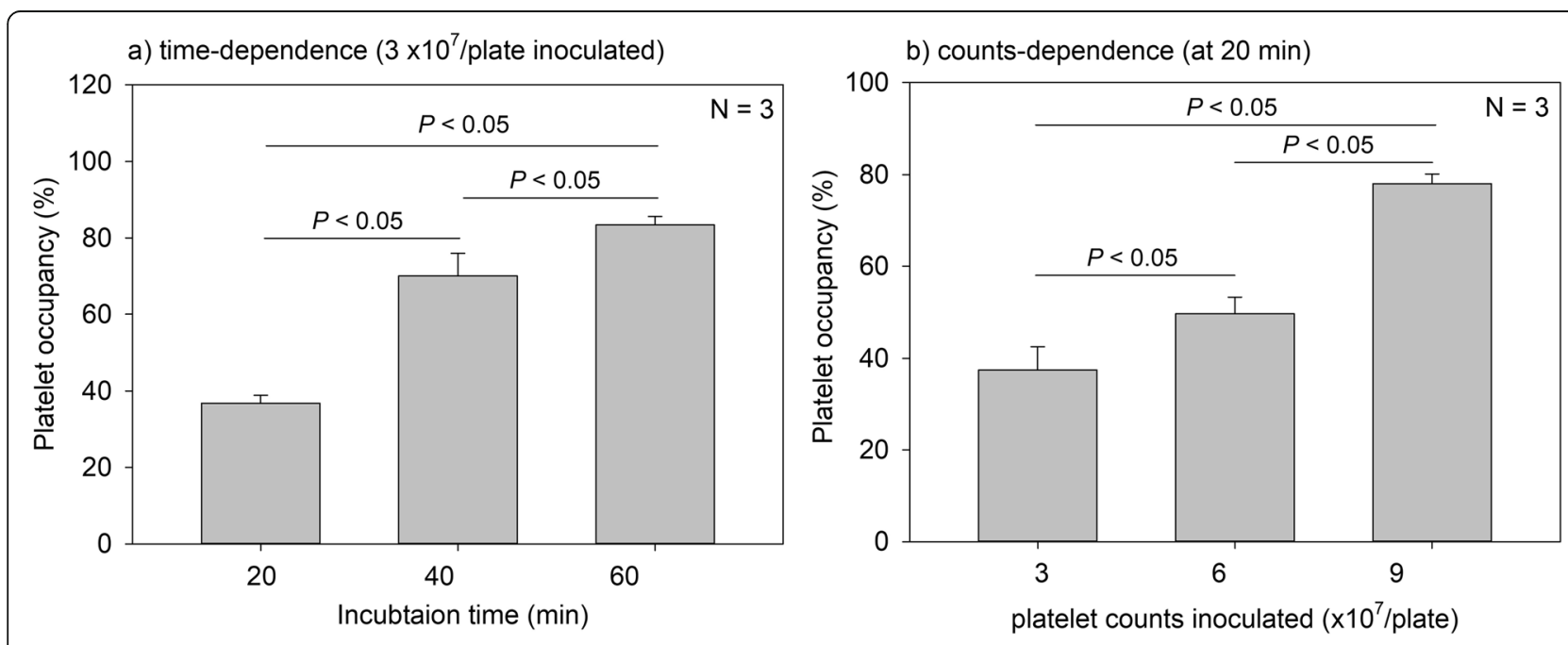

Fig. 6 Time- and count-dependent platelet occupancy on CaP-blasted cp-Ti plates. The images obtained by actin staining were subjected to image analysis and platelet occupancy against the fixed region of interest was calculated. $N=3$ 
a) Low magnification
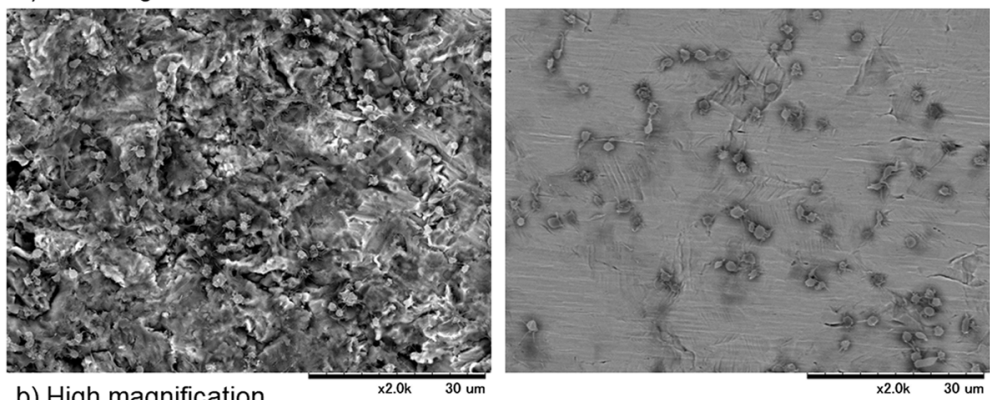

b) High magnification

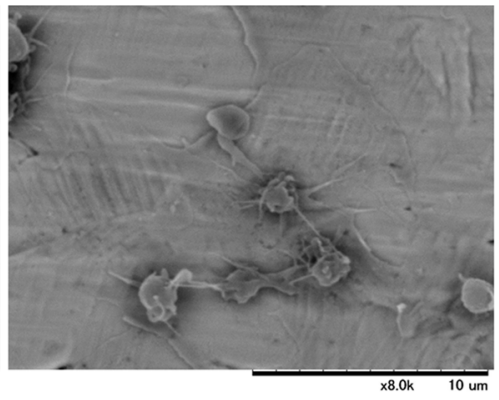

CaP-blasted Ti

plain SUS316L

Fig. 7 SEM observations of platelets adherent on CaP-blasted cp-Ti and plain SUS316L plates. PRP was placed on the plates, incubated for 60 min, washed, fixed, and dehydrated for SEM examination. a Low magnification and $\mathbf{b}$ high magnification

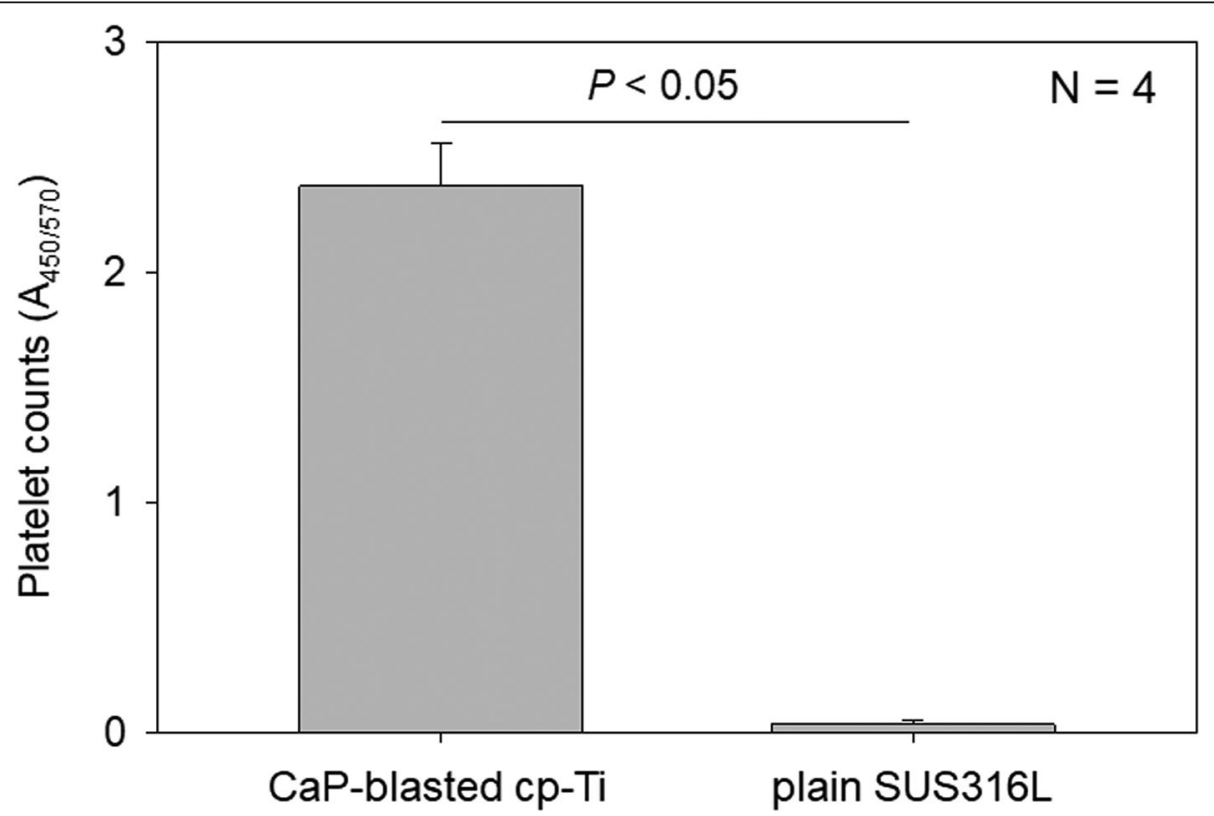

Fig. 8 Number of platelets adherent on CaP-blasted cp-Ti and plain SUS316L plates. After incubation for 60 min, the plates were rinsed and further incubated with CCK-8 for $90 \mathrm{~min}$. The amount of converted formazan was quantified using a microplate reader at $450 \mathrm{~nm}$. N=4.P<0.05 compared to SUS316 

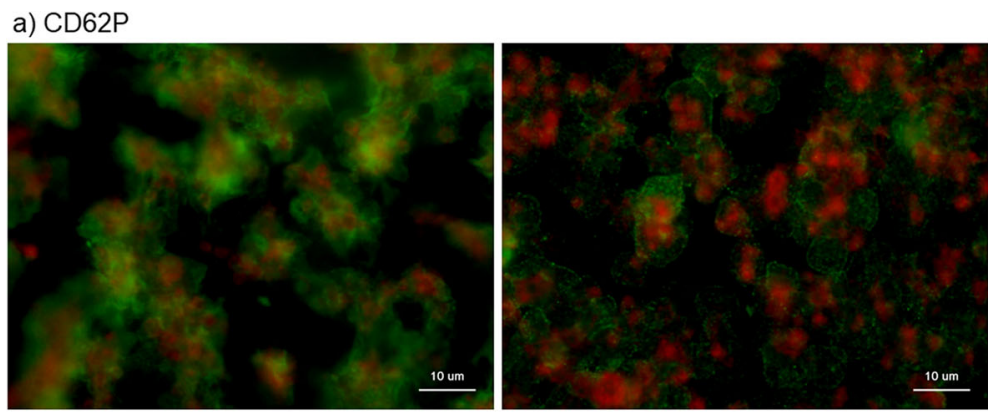

b) CD63
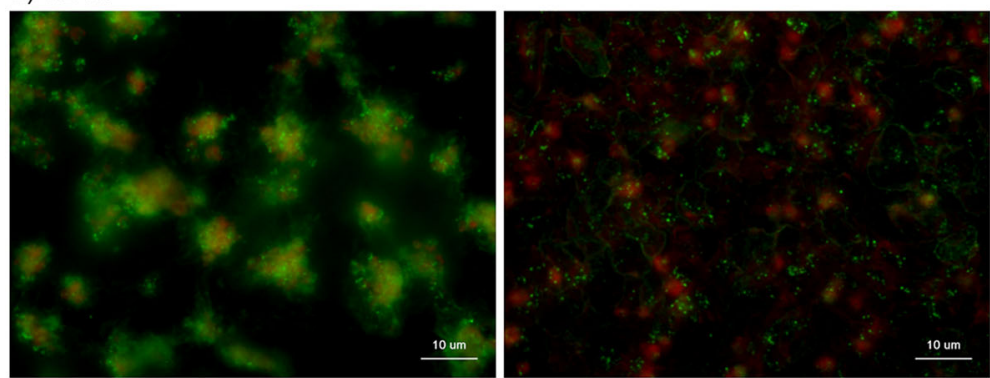

c) Isotype control
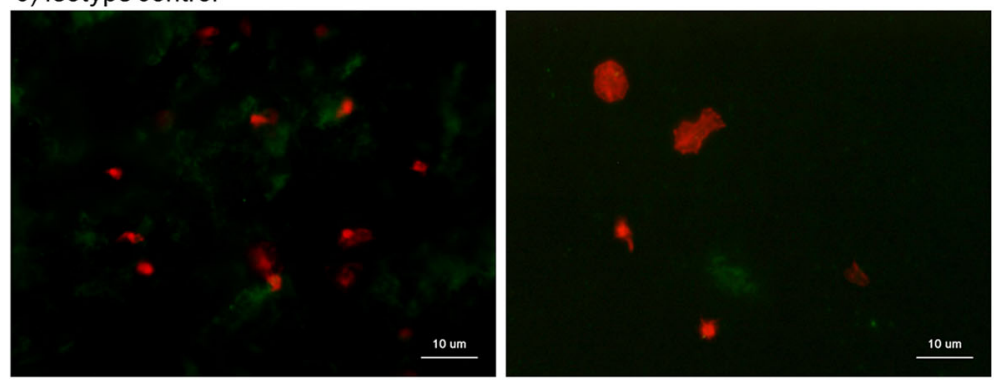

CaP-blasted cp-Ti

plain SUS316L

Fig. 9 Immunocytochemical detection of activated platelets expressing a CD62P or b CD63. CaP-blasted cp-Ti and plain SUS316L plates were incubated with PRP for $60 \mathrm{~min}$, washed, fixed, and subjected to immunocytochemical visualization. Green represents proteins reactive for a CD62P or b CD63, while red represents polymerized actin. $\mathbf{c}$ To show non-specific detection in extra-platelet spaces, PRP containing relatively low platelet counts was added on the plates and examined

PDGF-B-like proteins), which are stored in $\alpha$-granules [34], were localized mainly in platelets on the plain SUS316L surface. On the other hand, they were widely distributed in the extra-platelet spaces on the CaPblasted cp-Ti surface.

Polyphosphates are stored in dense granules and are released upon activation [35]. Thus, it is anticipated that similar to PDGF-B, polyphosphates would also be distributed in extra-platelet spaces upon activation. In this study, polyphosphates were visualized as particles in platelets on the plain SUS316L surface, and their distribution was similar to that of PDGF-B. In contrast, on the CaP-blasted cp-Ti surface, polyphosphates diffused, and thus faintly observed. Due to their nature, visualization and quantification of polyphosphates is not yet established, and is consequently limited, especially in terms of sensitivity [36]. However, recent advances have enabled the visualization of polyphosphates using DAPI, specifically by switching excitation and/or emission wavelengths [37]. We are still undergoing the process of further optimizing the experimental protocol; however, for the first time, we have successfully demonstrated that polyphosphates are released from activated platelets and diffuse, becoming diluted as they enter extra-platelet spaces.

It has generally been accepted that polyphosphates are involved in various platelet functions, such as coagulation [38]. In addition, their involvement in biomineralization has increasingly been focused on in the past decade [3943]. Therefore, to comprehensively understand the potential of PRP therapy, it is necessary to investigate the roles of polyphosphates more vigorously.

\section{SUS316L stainless steel plates}

SUS316L is a subtype of stainless steel that is widely used in medical devices, including injection needles. 

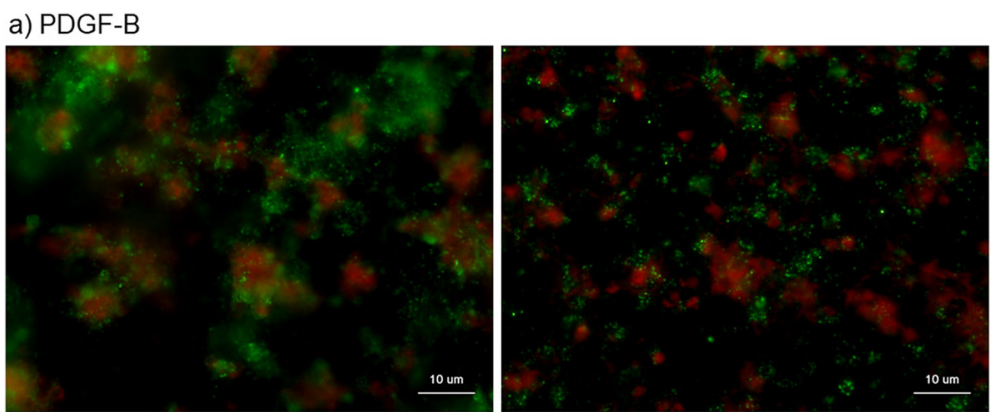

b) polyphosphates

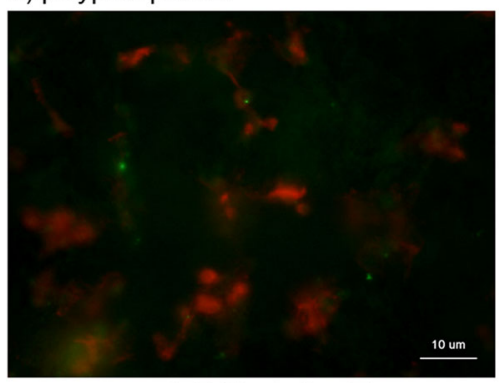

CaP-blasted cp-Ti

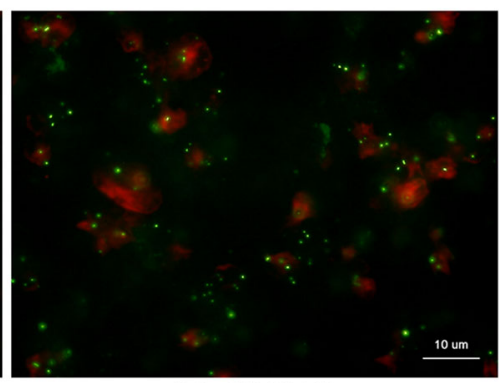

plain SUS316L

Fig. 10 a Immunocytochemical visualization of PDGF-B and $\mathbf{b}$ chemical detection of polyphosphates. CaP-blasted cp-Ti and plain SUS316 plates were incubated with PRP for 60 min, washed, fixed, and subjected to immunocytochemical or chemical detection. Green represents proteins reactive for PDGF-B (a) or polyphosphates $(\mathbf{b})$, while red represents polymerized actin

Laboratory technicians routinely use plastic pipettes for PRP preparation, while many clinicians and operators working in private practices prefer using syringes with injection needles, especially non-bevel needles. In the process of blood collection, the blood passes though these needles. It is believed that blood coagulation starts upon contact of platelets and coagulation factors with the inner walls of the needles when blood passes through them; thus, large bore needles are advised to be used to avoid unintentional activation of platelets [44, 45]. However, our present data suggest that the plain SUS316L surface may not significantly influence platelet activation, as long as relatively large bore needles are used. In addition, there may not be a significant loss in platelet levels in the presence of anticoagulants.

\section{Clinical relevance}

According to Davis [46, 47] and Junker et al. [10], among the series of events occurring between the host and the implant surface immediately after implant placement, the first and the most important healing phase is osteoconduction. This process promotes the directed growth of osteogenic cells through platelet activation, subsequent osteogenic cell migration, and fibrin clot stabilization on rough surface. Thus, we expected that this phase was facilitated or augmented by coating with the implant with PRP. Nikolidakis et al. [48, 49] demonstrated in an animal-based study that PRP in liquid form (without activation) increases bone apposition on $\mathrm{CaP}$ coated rough surface $\mathrm{Ti}$ implants. In this context, we suggest that the PRP immersion of CaP-blasted Ti implants prior to clinical application may be beneficial for initial implant stability.

To date, however, the subsequent preclinical and clinical studies have provided contradicting findings [50-54] and the effects of PRP remain controversial. Since implant osseointegration is influenced by several factors such as implant design, implant surface, surgical technique, bone type, and loading conditions in addition to the varied quality of individual PRP preparations [55], we should not conclude that PRP treatment does not benefit osseointegration or facilitate initial implant stability. In view of the current scenario, the European Association for Osseointegration, on its website, replies to clinicians' questions regarding the effects of PRP on implant success rates [56]. Neither PRP nor plasma-rich growth factors (PRGF) have been shown to improve implant stability or reduce marginal bone loss following implant placement. Although a randomized clinical trial was carried out, currently there is insufficient evidence to support a clinical recommendation on this basis [55].

This in vitro study focused on the effects of PRP immersion on implant surfaces using a small sample size $(N=6)$ by limiting sample donors, as followed in our previous studies $(N=2-15)$ in the past 2 years $[2,16$, $20,21,57-64]$. Thus, to evaluate the population type 
(Gaussian or not), individual differences, and spread of distribution, or to more strictly validate and generalize these results, further comparative studies with larger sample sizes are warranted that should include female donors.

\section{Conclusions}

Owing to their unique surface properties, CaP-blasted cp-Ti plates are capable of efficiently inducing platelet adhesion and activation to release biomolecules involved in regeneration of the surrounding tissue. Therefore, immersion of this type of dental implant in liquid PRP could contribute to the augmentation of their initial stability.

\section{Abbreviations}

PRP: Platelet-rich plasma; ACD-A: A formulation of acid-citrate-dextrose; $\mathrm{CP}$ Ti: Commercially pure titanium; PBS: Phosphate-buffered saline; HA: Hydroxyapatite; $\beta$-TCP: $\beta$-Tricalcium phosphate; CaP: Calcium phosphate; T-PBS: 0.1\% Tween-20-containing PBS; DAPI: 4',6-Diamidino-2-phenylindole

\section{Acknowledgements}

Not applicable.

\begin{abstract}
Authors' contributions
Conceptualization, MN and TK; methodology, TK; validation, $\mathrm{HA}, \mathrm{YK}$, and $\mathrm{TT}$; formal analysis, TW; investigation, MN, HA, HK, AS, TW, KI, and TK; data curation, $\mathrm{Kl}$ and $\mathrm{YK}$; writing-original draft preparation, $\mathrm{MN}, \mathrm{HA}, \mathrm{T}$, and TK; writing - review and editing, $\Pi \mathrm{T}$ and TK; supervision, YK; project administration, TW; funding acquisition, TK The authors have read and agreed to the published version of the manuscript.
\end{abstract}

\section{Funding}

This study was financially supported in part by JSPS KAKENHI (Grant Number 18K09595).

\section{Availability of data and materials}

The data are available from the corresponding author on reasonable request.

\section{Ethics approval and consent to participate}

The study design and consent forms of all the procedures performed were approved by the ethics committee for human participants of the Niigata University School of Medicine (Niigata, Japan) in accordance with the Helsinki Declaration of 1964 as revised in 2013. Written informed consents to participate in the study were obtained from all the participants.

\section{Consent for publication}

Written informed consent for the publication of these were obtained from all the participants.

\section{Competing interests}

Author MN, HA, HK, AS, TW, KI, YK, TT, and TK state that there are no conflicts of interest.

\section{Author details}

${ }^{1}$ Tokyo Plastic Dental Society, Kita-ku, Tokyo, Japan. ${ }^{2}$ Department of Materials Science and Technology, Niigata University, Niigata, Japan. ${ }^{3}$ Division of Oral Bioengineering, Institute of Medicine and Dentistry, Niigata University, Niigata, Japan

Received: 12 August 2020 Accepted: 25 October 2020

Published online: 20 November 2020

\section{References}

1. Kawase T. Platelet-rich plasma and its derivatives as promising bioactive materials for regenerative medicine: basic principles and concepts underlying recent advances. Odontology. 2015;103:126-35.
2. Takahashi A, Takahashi S, Tsujino T, Isobe K, Watanabe T, Kitamura Y, et al. Platelet adhesion on commercially pure titanium plates in vitro I: effects of plasma components and involvement of the von Willebrand factor and fibronectin. Int J Implant Dent. 2019:5:5.

3. Hanawa T. Biofunctionalization of titanium for dental implant. Jpn Dent Sci Rev. 2010;46:93-101.

4. Ishikawa K, Miyamoto Y, Nagayama M, Asaoka K. Blast coating method: new method of coating titanium surface with hydroxyapatite at room temperature. J Biomed Mater Res. 1997;38:129-34.

5. Watanabe T, Nakagawa E, Saito K, Ohshima H. Differences in healing patterns of the bone-implant interface between immediately and delayedplaced titanium implants in mouse maxillae. Clin Implant Dent Relat Res. 2016:18:146-60.

6. Makishi S, Saito K, Ohshima H. Osteopontin-deficiency disturbs direct osteogenesis in the process of achieving osseointegration following immediate placement of endosseous implants. Clin Implant Dent Relat Res. 2017;19:496-504.

7. Civantos A, Martínez-Campos E, Ramos V, Elvira C, Gallardo A, Abarrategi A. Titanium coatings and surface modifications: toward clinically useful bioactive implants. ACS Biomaterials Science \& Engineering. 2017;3:1245-61.

8. Arcos D, Vallet-Regí M. Substituted hydroxyapatite coatings of bone implants. J Mater Chem B. 2020;8:1781-800.

9. de Groot K, Wolke JG, Jansen JA. Calcium phosphate coatings for medical implants. Proc Inst Mech Eng H. 1998;212:137-47.

10. Junker $R$, Dimakis $A$, Thoneick $M$, Jansen JA. Effects of implant surface coatings and composition on bone integration: a systematic review. Clin Oral Implants Res. 2009;20(Suppl 4):185-206.

11. Kawase T, Tanaka T, Minbu H, Kamiya M, Oda M, Hara T. An atmosphericpressure plasma-treated titanium surface potentially supports initial cell adhesion, growth, and differentiation of cultured human prenatal-derived osteoblastic cells. J Biomed Mater Res B Appl Biomater. 2014;102:1289-96.

12. Goto T, Yoshinari M, Kobayashi S, Tanaka T. The initial attachment and subsequent behavior of osteoblastic cells and oral epithelial cells on titanium. Biomed Mater Eng. 2004;14:537-44.

13. Knabe C, Howlett CR, Klar F, Zreiqat $H$. The effect of different titanium and hydroxyapatite-coated dental implant surfaces on phenotypic expression of human bone-derived cells. J Biomed Mater Res A. 2004;71:98-107.

14. Lumbikanonda N, Sammons R. Bone cell attachment to dental implants of different surface characteristics. Int J Oral Maxillofac Implants. 2001; 16:627-36.

15. Damiati L, Eales MG, Nobbs AH, Su B, Tsimbouri PM, Salmeron-Sanchez M, et al. Impact of surface topography and coating on osteogenesis and bacterial attachment on titanium implants. J Tissue Eng. 2018;9: 2041731418790694

16. Tsujino T, Takahashi A, Watanabe T, Isobe K, Kitamura Y, Okuda K, et al. Platelet adhesion on commercially pure titanium plates in vitro II. Immunofluorescence visualization of PDGF-B, TGF $\beta 1$, and PPARY released from activated adherent platelets. Dent J (Basel). 2019;7:109.

17. Jeong J, Kim JH, Shim JH, Hwang NS, Heo CY. Bioactive calcium phosphate materials and applications in bone regeneration. Biomaterials Research. 2019;23:4.

18. Surmenev RA, Surmeneva MA, Ivanova AA. Significance of calcium phosphate coatings for the enhancement of new bone osteogenesis--a review. Acta Biomater. 2014;10:557-79.

19. Kobayashi M, Kawase T, Horimizu M, Okuda K, Wolff LF, Yoshie H. A proposed protocol for the standardized preparation of PRF membranes for clinical use. Biologicals. 2012:40:323-9.

20. Tsujino T, Takahashi A, Yamaguchi S, Watanabe T, Isobe K, Kitamura Y, et al. Evidence for contamination of silica microparticles in advanced platelet-rich fibrin matrices prepared using silica-coated plastic tubes. Biomedicines. 2019;7:45.

21. Masuki H, Isobe K, Kawabata H, Tsujino T, Yamaguchi S, Watanabe T, et al. Acute cytotoxic effects of silica microparticles used for coating of plastic blood-collection tubes on human periosteal cells. Odontology. 2020. https:// doi.org/10.1007/s10266-020-00486-z, https://doi.org/10.1007/s10266-02000486-z.

22. Toyoda T, Isobe $K$, Tsujino T, Koyata $Y$, Ohyagi $F$, Watanabe $T$, et al. Direct activation of platelets by addition of $\mathrm{CaCl} 2$ leads coagulation of platelet-rich plasma. Int J Implant Dent. 2018:4:23.

23. Kim I-H, Kwon T-Y, Kim K-H. Wetting behavior of dental implants. In: Aliofkhazraei M, editor. Wetting and wettability. London: IntechOpen; 2015. p. 253-70. 
24. Zeng H, Chittur KK, Lacefield WR. Analysis of bovine serum albumin adsorption on calcium phosphate and titanium surfaces. Biomaterials. 1999; 20:377-84

25. Robotti F, Bottan S, Fraschetti F, Mallone A, Pellegrini G, Lindenblatt N, et al. A micron-scale surface topography design reducing cell adhesion to implanted materials. Sci Rep. 2018;8:10887.

26. Toriyama M, Kawamoto Y, Suzuki T, Yokogawa Y, Nishizawa K, Nagata F. Wettability of calcium phosphate ceramics by water. J Ceram Soc Jpn. 1995; 103:46-9.

27. Zahran R, Rosales Leal Jl, Rodríguez Valverde MA, Cabrerizo Vílchez MA. Effect of hydrofluoric acid etching time on titanium topography, chemistry, wettability, and cell adhesion. PLoS One. 2016;11:e0165296.

28. Kubiak KJ, Wilson MCT, Mathia TG, Carval P. Wettability versus roughness of engineering surfaces. Wear. 2011;271:523-8.

29. dos Santos EA, Farina M, Soares GA, Anselme K. Surface energy of hydroxyapatite and beta-tricalcium phosphate ceramics driving serum protein adsorption and osteoblast adhesion. J Mater Sci Mater Med. 2008; 19:2307-16

30. Zhu XD, Zhang HJ, Fan HS, Li W, Zhang XD. Effect of phase composition and microstructure of calcium phosphate ceramic particles on protein adsorption. Acta Biomater. 2010;6:1536-41.

31. Ong JL, Chittur KK, Lucas LC. Dissolution/reprecipitation and protein adsorption studies of calcium phosphate coatings by FT-IR/ATR techniques. J Biomed Mater Res. 1994;28:1337-46.

32. Villarreal DR, Sogal A, Ong JL. Protein adsorption and osteoblast responses to different calcium phosphate surfaces. J Oral Implantol. 1998;24:67-73.

33. Kon E, Filardo G, Di Martino A, Marcacci M. Platelet-rich plasma (PRP) to treat sports injuries: evidence to support its use. Knee Surg Sports Traumatol Arthrosc. 2011;19:516-27.

34. Blair P, Flaumenhaft R. Platelet alpha-granules: basic biology and clinical correlates. Blood Rev. 2009;23:177-89.

35. Ruiz FA, Lea CR, Oldfield E, Docampo R. Human platelet dense granules contain polyphosphate and are similar to acidocalcisomes of bacteria and unicellular eukaryotes. J Biol Chem. 2004:279:44250-7.

36. Mailer RKW, Hänel L, Allende M, Renné T. Polyphosphate as a target for interference with inflammation and thrombosis. Frontiers in Medicine. 2019;6:76.

37. Martin P, Van Mooy BAS. Fluorometric quantification of polyphosphate in environmental plankton samples: extraction protocols, matrix effects, and nucleic acid interference. Appl Environ Microbiol. 2013;79:273-81.

38. Travers RJ, Smith SA, Morrissey JH. Polyphosphate, platelets, and coagulation. Int J Lab Hematol. 2015;37(Suppl 1):31-5.

39. St-Pierre JP, Pilliar RM, Grynpas MD, Kandel RA. Calcification of cartilage formed in vitro on calcium polyphosphate bone substitutes is regulated by inorganic polyphosphate. Acta Biomater. 2010;6:3302-9.

40. Hoac B, Kiffer-Moreira T, Millán JL, McKee MD. Polyphosphates inhibit extracellular matrix mineralization in MC3T3-E1 osteoblast cultures. Bone. 2013;53:478-86.

41. Li L, Khong ML, Lui ELH, Mebarek S, Magne D, Buchet R, et al. Long-chain polyphosphate in osteoblast matrix vesicles: enrichment and inhibition of mineralization. Biochim Biophys Acta Gen Subj. 1863;2019:199-209.

42. Omelon S, Georgiou J, Henneman ZJ, Wise LM, Sukhu B, Hunt T, et al. Control of vertebrate skeletal mineralization by polyphosphates. PLoS One. 2009;4:e5634.

43. Wang Y, Li M, Li P, Teng H, Fan D, Du W, et al. Progress and applications of polyphosphate in bone and cartilage regeneration. Biomed Res Int. 2019; 2019:5141204

44. Harmon K, JHanson R, Bowen J, Greenberg S, Magaziner E, Vandenbosch J, Audley D. et al.. Guidelines for the use of platelet rich plasma.

45. Peterson NS, Reeves KD. Efficacy of one day training in low-cost manual preparation of high cellular platelet rich plasma. J Prolotherapy. 2014;6:e922-e27.

46. Davies JE. Understanding peri-implant endosseous healing. J Dent Educ. 2003;67:932-49.

47. Davies JE. Bone bonding at natural and biomaterial surfaces. Biomaterials. 2007:28:5058-67.

48. Nikolidakis D, van den Dolder J, Wolke JG, Jansen JA. Effect of platelet-rich plasma on the early bone formation around Ca-P-coated and non-coated oral implants in cortical bone. Clin Oral Implants Res. 2008;19:207-13.

49. Nikolidakis D, van den Dolder J, Wolke JG, Stoelinga PJ, Jansen JA. The effect of platelet-rich plasma on the bone healing around calcium phosphate-coated and non-coated oral implants in trabecular bone. Tissue Eng. 2006;12:2555-63.
50. Attia S, Narberhaus C, Schaaf H, Streckbein P, Pons-Kühnemann J, Schmitt C, et al. Long-term influence of platelet-rich plasma (PRP) on dental implants afte maxillary augmentation: implant survival and success rates. J Clin Med. 2020;9: 391.

51. Birang $R$, Tavakoli M, Shahabouei M, Torabi A, Dargahi A, Soolari A. Investigation of peri-implant bone healing using autologous plasma rich in growth factors in the canine mandible after 12 weeks: a pilot study. Open Dent J. 2011;5:168-73.

52. Kundu R, Rathee M. Effect of platelet-rich-plasma (PRP) and implant surface topography on implant stability and bone. J Clin Diagn Res. 2014;8:Zc26-30

53. Monov G, Fuerst G, Tepper G, Watzak G, Zechner W, Watzek G. The effect of platelet-rich plasma upon implant stability measured by resonance frequency analysis in the lower anterior mandibles. Clin Oral Implants Res. 2005; 16:461-5.

54. Yun JH, Han SH, Choi SH, Lee MH, Lee SJ, Song SU, et al. Effects of bone marrow-derived mesenchymal stem cells and platelet-rich plasma on bone regeneration for osseointegration of dental implants: preliminary study in canine three-wall intrabony defects. J Biomed Mater Res B Appl Biomater. 2014:102:1021-30.

55. Kawase T, Mubarak S, Mourão CF. The platelet concentrates therapy: from the biased past to the anticipated future. Bioengineering (Basel). 2020;7:82

56. European Association for Osseointegration. Platelet concentrates. "Key points for Clinical Practice from the EAO Consensus Conference".

57. Aizawa H, Kawabata H, Sato A, Masuki H, Watanabe T, Tsujino T, et al. A comparative study of the effects of anticoagulants on pure platelet-rich plasma quality and potency. Biomedicines. 2020:8:42

58. Aizawa H, Tsujino T, Watanabe T, Isobe K, Kitamura Y, Sato A, et al. Quantitative near-infrared imaging of platelets in platelet-rich fibrin (PRF) matrices: comparative analysis of bio-PRF, leukocyte-rich PRF, advanced-PRF and concentrated growth factors. International Jounral of Molecular Sicences. 2020;21:4426

59. Yamaguchi S, Aizawa H, Sato A, Tsujino T, Isobe K, Kitamura Y, et al. Concentrated growth factor matrices prepared using silica-coated plastic tubes are distinguishable from those prepared using glass tubes in platelet distribution: application of a novel near-infrared imaging-based, quantitative technique. Front Bioeng Biotechnol. 2020;8:600.

60. Tsujino T, Masuki H, Nakamura M, Isobe K, Kawabata H, Aizawa H, et al. Striking differences in platelet distribution between advanced-platelet-rich fibrin and concentrated growth factors: effects of silica-containing plastic tubes. Journal of Functional Biomaterials. 2019;10:43.

61. Tsujino T, Isobe K, Kawabata H, Aizawa H, Yamaguchi S, Kitamura Y, et al. Spectrophotometric determination of the aggregation activity of platelets in platelet-rich plasma for better quality control. Dent J (Basel). 2019;7:61.

62. Takahashi A, Tsujino T, Yamaguchi S, Isobe K, Watanabe T, Kitamura Y, et al. Distribution of platelets, TGF $\beta 1$, PDGF-BB, VEGF, MMP9 and fibronectin in advanced platelet-rich fibrin (A-PRF) and concentrated growth factors (CGF) matrices. J Investig Clin Dent. 2019;10:e12458.

63. Kawase T, Nagata M, Okuda K, Ushiki T, Fujimoto $Y$, Watanabe $M$, et al. Plateletrich fibrin extract: a promising fetal bovine serum alternative in explant cultures of human periosteal sheets for regenerative therapy. Int J Mol Sci. 2019;20:1053.

64. Kitamura Y, Suzuki M, Tsukioka T, Isobe K, Tsujino T, Watanabe T, et al. Spectrophotometric determination of platelet counts in platelet-rich plasma. Int J Implant Dent. 2018;4:29.

\section{Publisher's Note}

Springer Nature remains neutral with regard to jurisdictional claims in published maps and institutional affiliations. 